\title{
COMPARISON OF VISUAL ASSESSMENT TESTS IN MULTIPLY HANDICAPPED CHILDREN
}

\author{
R. T. MACKIE ${ }^{1}$, D. L. McCULLOCH ${ }^{1}$, K. J. SAUNDERS ${ }^{1}$, J. BALLANTYNE ${ }^{1}$, R. E. DAY ${ }^{2}$, \\ M. S. BRADNAM ${ }^{2}$ and G. N. DUTTON ${ }^{2}$ \\ Glasgow
}

\begin{abstract}
SUMMARY
The aims of this study were to compare acuity estimates achieved with visual evoked potential (VEP) and acuity card techniques and to examine the success rates of each test in a group of multiply handicapped children. Subjects were 52 children (3-183 months) with multiple handicaps associated with prematurity $(n=17)$, congenital anomalies $(n=16)$, hypoxic insult $(n=10)$ and other disorders $(n=9)$. Success rates for completing the tests were: VEP $88 \%$ and acuity cards $85 \%$ (Keeler or Cardiff). The acuity card tests were less likely to be successfully completed in the severely disabled $(p<0.05)$ and in those children with nystagmus $(p<0.05)$. When both acuity cards were successful, results agreed to within \pm 1.75 octaves. Acuity card thresholds were significantly correlated with VEP thresholds $(p<0.02)$, but thresholds achieved with VEPs were better in children with poor vision.
\end{abstract}

Accurate assessment of vision in multiply handicapped children is difficult due to problems arising from impaired communication, and the physical demands of test procedures. Many of these children are non-verbal due to intellectual impairment or motor disability, and their comprehension is limited. Those with the most severe neurological disorders are more likely to have severe visual impairment. ${ }^{1-4}$ Visual impairment may be a result of refractive error $\left(23 \%{ }^{5}-64 \%^{6}\right)$, disorders of muscle balance (strabismus $29 \%{ }^{5}-69 \%{ }^{6}$, nystagmus $6 \%^{7}-18 \%{ }^{6}$ ), pathology of the globe (cataract $4 \%^{5}-27 \%^{8}$, optic atrophy $1 \%{ }^{9}-10 \%{ }^{10}$, optic nerve hypoplasia) or cortical damage (visual field defects $11 \%^{10}$, cortical blindness $3.1 \%^{11}$ ).

Previous studies on visual assessment of multiply handicapped children have used methods initially developed for the testing of infants. These methods

From: ${ }^{1}$ Glasgow Caledonian University; ${ }^{2}$ Royal Hospital for Sick Children, Glasgow, UK.

Correspondence to: Roisin T. Mackie, Department of Vision Sciences, Glasgow Caledonian University, Cowcaddens Road, Glasgow G4 0BA, UK. include preferential looking (PL) and visual evoked potentials (VEP). Early studies used forced-choice PL with good success rates $(84 \%-92 \%),{ }^{12-17}$ but the increased use of the acuity card procedure has improved testability and the accuracy of acuities reported. This technique is a more rapid and flexible adaptation of PL, and is obviously more suited to the diversity of ability encountered in the handicapped population. Results show that the more severely handicapped children have the greatest impairment of vision; ${ }^{4,17}$ acuities reported range from 6.9 to 0.3 cycles/degrees. ${ }^{18}$ Mohn and van Hof-van Duin ${ }^{17,19}$ reported that mildly handicapped children demonstrated acuities from normal to within 1 octave of normal, with the acuity inversely related to severity of intellectual impairment. An octave is equal to a doubling of the pattern size.

Several studies have used VEPs to assess visual function in multiply handicapped populations. OrelBixler and colleagues ${ }^{4}$ used a swept spatial frequency stimulus on 59 multiply and severely handicapped patients (3-33 years). Their success in obtaining VEP acuity estimates was high $(95 \%)$, and when compared with acuity card thresholds there was agreement to within 1 octave in $66 \%$ of cases. When agreement was outside these limits, the VEP measure was generally higher than the acuity card measure. However, in a small sample of handicapped patients, Mohn and van Hof-van Duin ${ }^{20}$ reported VEP acuities determined with pattern reversal stimulus to be lower than acuity card acuities.

The present study investigated the use of VEPs and acuity cards in a group of multiply handicapped children with a wide range of handicaps and abilities. The degree of successful completion of both test procedures and a comparison between acuity measures obtained is reported.

\section{SUBJECTS AND METHODS}

Subjects

Subjects were 52 children (3-183 months) with 
Table 1. Aetiology of handicaps

\begin{tabular}{llc}
\hline Aetiology & Subgroup & Number \\
\hline Ischaemic insult & Perinatal ischaemia & 5 \\
& Postnatal ischaemia & 5 \\
Prematurity & Intracranial haemorrhage & 3 \\
(associated with) & Periventricular leucomalacia & 3 \\
& Hypoxia & 1 \\
& Septicaemia & 1 \\
& Unknown factors & 9 \\
Congenital & Hydrocephalus & 3 \\
& Microcephaly & 3 \\
& Lissencephaly & 2 \\
& Metabolic & 2 \\
& Retts syndrome & 2 \\
& Rubella syndrome & 1 \\
& Down's syndrome & 1 \\
Infectious & Hypotonia & 1 \\
Unknown & Encephalocoele & 1 \\
& Meningitis & 3 \\
& & 6 \\
\hline
\end{tabular}

multiple handicaps with uncertain, or unreliable, estimates of visual function after previous eye or neurological examinations. Significant refractive errors were corrected prior to acuity testing.

Aetiology was diverse, with congenital abnormalities (31\%) and complications of prematurity (33\%) forming the largest groups (Table I). A small number of children $(12 \%)$ had unknown causes of handicap. All children had a combination of physical and/or intellectual impairment. Intellectual impairment was ranked from normal to severe disability by a paediatric neurologist (R.D.), who used a review of previous medical records and performed a neurological examination. Distribution of levels of intellectual impairment was surprisingly even (normal $27.7 \%$; mild $21.3 \%$; moderate $25.5 \%$; severe $25.5 \%$ ).

\section{Methods}

VEP and acuity card testing were performed on the same day in random order, with the examiners masked to the previous test results.

VEP Method. Binocular VEPs were recorded to the onset of black and white stimulus patterns from a luminance matched grey (pattern onset VEP). Patterns were presented repetitively at $1.06 \mathrm{~Hz}$ and the signals were averaged 50 times to produce a VEP waveform. Signals were recorded from an active occipital electrode $\left(\mathrm{O}_{\mathrm{z}}\right)$ referenced and grounded to the earlobes. An artefact rejection program eliminated trials with amplitudes exceeding $\pm 50 \mu \mathrm{V}$, which would occur due to excessive movement of the patient, or background EEG activity-both frequent occurrences during testing. Viewing distance was either $43 \mathrm{~cm}$ or $85 \mathrm{~cm}$ depending on the abilities and age of the child.

Stimulus patterns used were checks subtending 120 minutes of arc, and vertical gratings of various spatial frequencies. The grating stimuli moved horizontally at 14 cycles/s or 3 cycles/s for $200 \mathrm{~ms}$ after onset. For both types of stimuli each presentation lasted $200 \mathrm{~ms}$. Black and white stimuli were generated on a colour monitor with a mean luminance of $90 \mathrm{~cd} / \mathrm{m}^{2}$, and a contrast between the black and white stripes of at least $90 \%$. The first stimulus presented was the $120^{\prime}$ stationary chequerboard. The response to this determined whether to use larger or smaller stimulus size to find threshold, by means of a staircase presentation. The minimum size stimulus which produced a waveform with a measurable positive peak (P1), and which was reproducible to within $\pm 20 \mathrm{~ms}$, was regarded as threshold. ${ }^{21}$ The P1 was defined as the largest peak within a time window of $50-150 \mathrm{~ms}$. Fig. 1 shows examples of VEP waveforms to supra- and infra-threshold stimuli. The VEP to $12^{\prime}$ bars is clearly not reproduced and is judged to be below threshold.

Testing was performed in a darkened, quiet room to provide minimum distraction. The child's attention was maintained by talking, rattling keys or singing. Fixation was monitored and recording was paused when the child looked away.

PL Method. Acuity cards used were Keeler Acuity

1. Supra-threshold VEPs to 60' bars

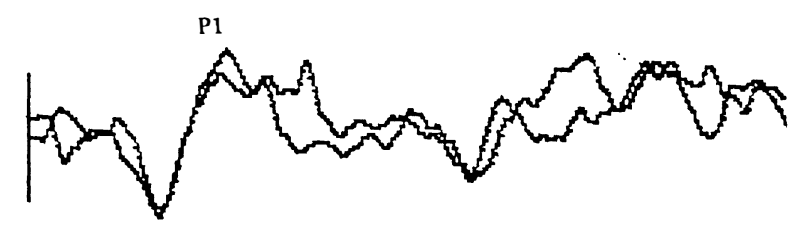

2. Threshold VEPs to 30' bars

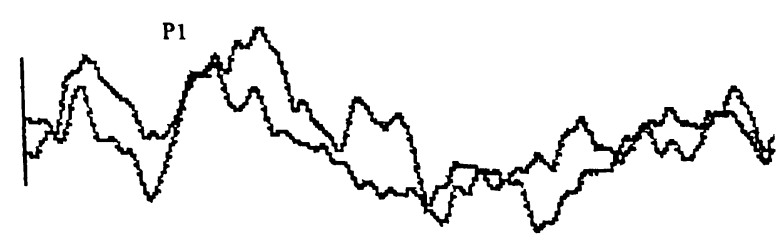

3. Infra-threshold VEPs to 12' bars

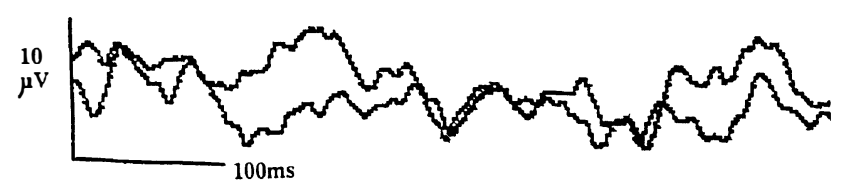

Fig. 1. VEPs recorded at $O_{z}$ to 50 presentations in a 14year-old multiply handicapped girl with moderate intellectual impairment. Supra-threshold VEPs (60' gratings) show good reproducibility of the P1. Threshold VEPs (30' gratings) show reproducibility of the P1 to within $20 \mathrm{~ms}$. Infra-threshold VEPs show no distinctive P1 and no reproducibility. 
Cards and the Cardiff Acuity Test. ${ }^{22}$ Keeler cards have a high-contrast black and white grating on one side of each card, the other side being a homogeneous field of matched luminance. When the spatial frequency of the grating presented cannot be resolved it matches the homogeneous field. These cards differ from Teller Acuity Cards in that the grating and the homogeneous field are both contained within a white circular border. This removes the edge artefact present with Teller cards which enables the square edge of the grating to be seen when the grating itself is beyond recognition. ${ }^{23}$ The cards may be presented either horizontally or vertically and are thus useful when examining children with horizontal nystagmus or hemianopia. The stimuli on Cardiff cards are familiar black and white pictures (e.g. car, house, fish) presented on a grey background. The pictures are 'vanishing optotypes' such that the average luminance of the black and white of the picture is equal to that of the neutral grey background. When the picture cannot be resolved it 'disappears' into the background. The cards are designed to be presented vertically only.

Due to the range of abilities and acuities encountered in the present study, both sets of cards were used in random order. Starting with a low spatial frequency, the examiner presented the cards to the child and judged by eye and head movements whether they could see the stimulus. Pointing to the stimulus, or naming it (Cardiff cards), was encouraged to help maintain attention with the more able children. By means of a staircase presentation the threshold size was estimated in a short space of time.

\section{RESULTS}

\section{Success Rates}

Of the 43 children on whom acuity card testing was attempted, $85 \%$ successfully completed at least one set of cards, resulting in a reliable acuity estimate. Failure to achieve an acuity estimate was due to patient non-compliance, or difficulty in assessing looking behaviour in the presence of abnormal eye movements or motor impairment. Twenty-five children completed both sets of cards; 7 of these cooperated with the testing procedure but failed to demonstrate looking behaviour in response to the largest card in both sets and were deemed to have minimal visual function. Their results are excluded from further analysis.

VEPs were attempted in 48 children with a success rate of $95 \%$. This includes 4 children for whom testing was judged to be a partial success. This occurred when the child co-operated sufficiently for the test to be completed but the VEP quality was poor due to recording difficulties or the presence of seizure activity. This resulted in an estimate of

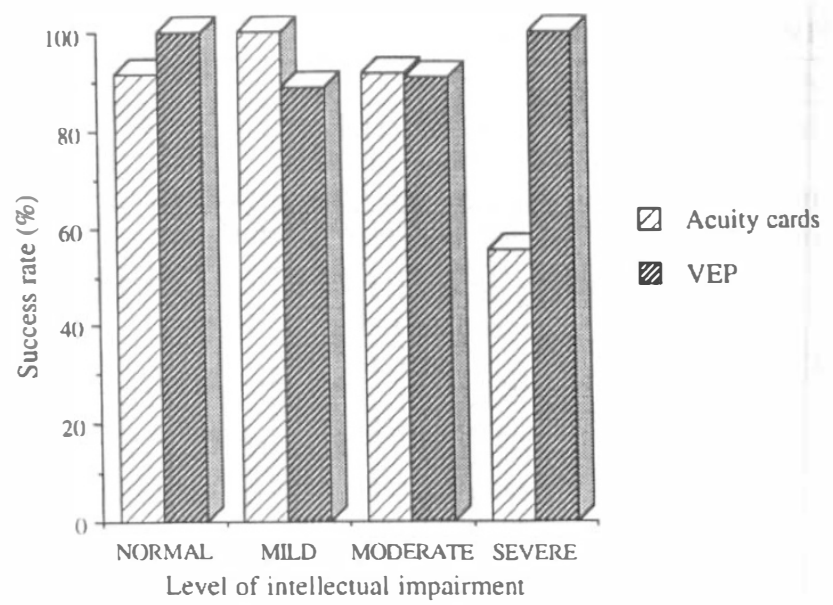

Fig. 2. Success rates for completing acuity cards and VEPS according to the level of intellectual impairment. Acuity cards were considered successful if either the Keeler or the Cardiff cards produced a reliable acuity estimate. VEP success rates include 4 children who completed testing but had poor-quality VEPs due to recording difficulties.

'minimal acuity'. Failure to complete testing was solely due to non-compliance.

The success rates of the acuity cards and VEPs were related to age, level of intellectual impairment, and the presence or absence of strabismus, nystagmus or field defect using a three-way analysis of variance (ANOVA). VEP success rates were not significantly different in any of these subgroups, but the acuity cards were less likely to be successfully completed in the severely intellectually impaired $(p<0.05$, Fisher PLSD) and in those children with nystagmus $(p<0.05$, Fisher PLSD). Fig. 2 shows the success rates of the tests for each level of intellectual impairment.

\section{Acuity Thresholds}

For comparisons between the different tests, thresholds are expressed as octave loss relative to a threshold of 30 cycles/degree ( $1^{\prime}$ bars).

Keeler Cards versus Cardiff Cards. Fig. 3 shows a linear regression relating acuity thresholds estimated by Keeler and Cardiff cards in those children who completed both tests. The graph shows a close correlation $\left(p=0.0001, r^{2}=0.68\right)$. This relationship was examined further by plotting the difference between the two thresholds estimates against the mean of the thresholds (Fig. 4). This graph provides information on the level of agreement between the two techniques and the presence or absence of bias. Fig. 4 shows close agreement between the card thresholds. The limits of agreement (mean $\pm 2 \mathrm{SD}$ ) shown on the graph are \pm 1.75 octaves. The scatter of the differences shows that the acuity level does not bias the agreement between the two acuity card tests. VEP versus Acuity Cards. We found no statistical difference between the thresholds achieved between 


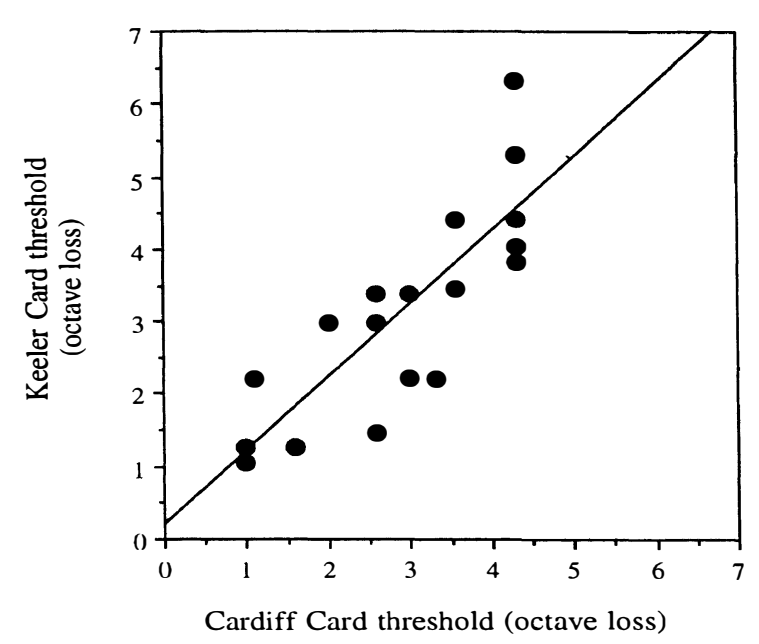

Fig. 3. A comparison of Cardiff card and Keeler card acuities shows good correlation between the acuity thresholds $\left(\mathrm{y}=0.19+1.0 \mathrm{x}, \mathrm{r}^{2}=0.681\right)$. Thresholds were expressed as octave loss relative to a threshold of 30 cycles/degree (1' bars).

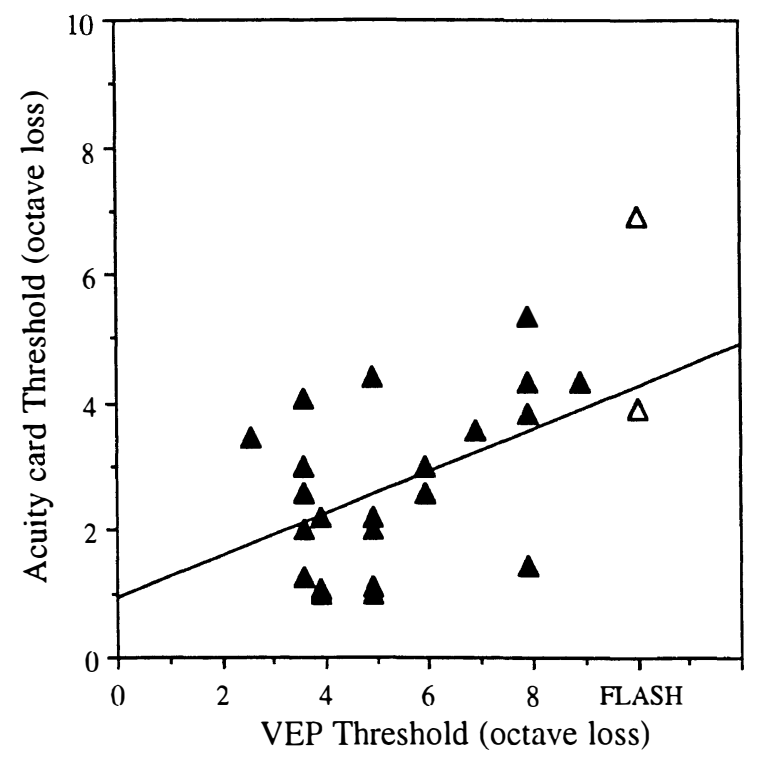

Fig. 5. A comparison of $V E P$ and acuity card acuities shows a significant correlation, but VEP acuities are lower than acuity card acuities in the majority of cases $(\mathrm{y}=0.71+$ $\left.0.4 \mathrm{x}, \mathrm{r}^{2}=0.34\right)$. The empty triangles indicate the children with only flash VEP responses; these points are not included in the analysis. Thresholds were expressed as octave loss relative to a threshold of 30 cycles/degree ( $1^{\prime}$ bars).

Keeler cards and Cardiff cards $(p=0.46$, Student's $t$ test). Therefore, VEP thresholds were compared with the best acuity estimate from the two sets of cards in those children who completed both sets, and the only result in the remaining children. One child aged 18 months was excluded from further analysis as VEP and acuity card thresholds develop at

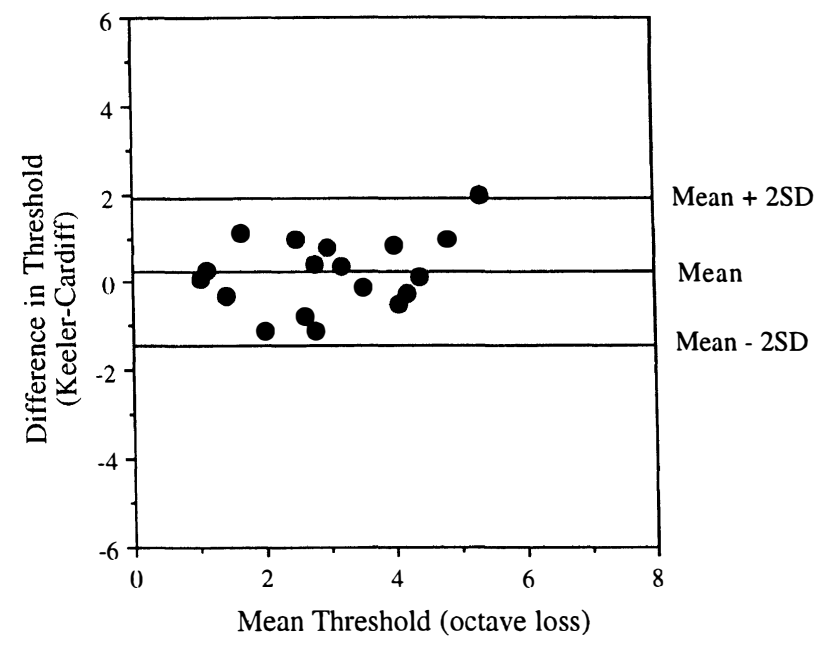

Fig. 4. The difference between Keeler card and Cardiff card acuity thresholds versus the mean acuity threshold. The scatter of differences shows good agreement between the acuity thresholds and the limits of agreement shown are narrow ( \pm 1.75 octaves).

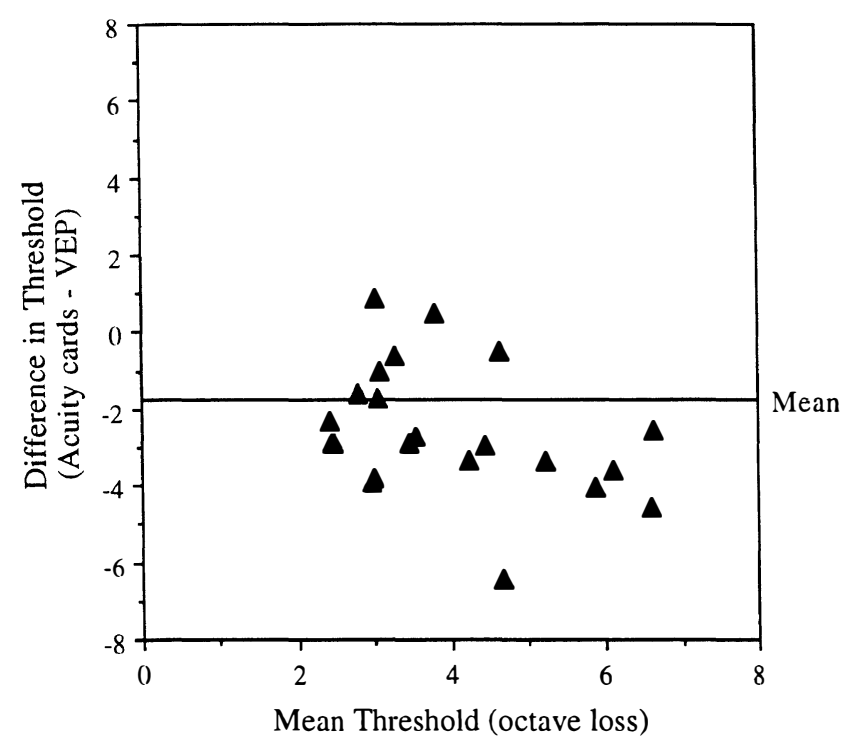

Fig. 6. The difference between acuity card and VEP acuity thresholds versus the mean acuity threshold. The scatter of differences shows bias between the two acuity thresholds, indicating that VEP acuities are higher in children with poor vision (>4 octave loss) relative to the acuity card acuities.

different rates in young children. ${ }^{24} \mathrm{~A}$ further 2 children who demonstrated only a flash VEP response were also excluded because an acuity threshold could not be estimated from this. Using linear regression analysis (Fig. 5) VEP and acuity card thresholds were significantly correlated $\left(p<0.02, r^{2}=0.34\right)$, but the slope of the graph 
indicates that VEP estimates were lower than acuity card estimates in the majority of cases. Plotting the difference between the two thresholds estimates against the mean of the thresholds (Fig. 6) shows moderate agreement between the two methods, but there is bias present. Children with poor vision $(>4$ octave loss) demonstrate higher VEP estimates relative to acuity card estimates. This bias was not demonstrated by subjects with higher levels of acuity.

\section{DISCUSSION}

The use of acuity cards and VEPs in handicapped children has enabled previously untested children to have a visual acuity assessment. In this study acuity cards were successfully used in children with mild to moderate levels of intellectual impairment, but were less successful in those who were severely intellectually impaired. This is because acuity determination is made from a behavioural response to the stimulus. Many severely and profoundly handicapped children do not respond to external stimulus and are very withdrawn. The good success rates for VEPs in this group are possible because no response is required from the patients. Although the VEPs tend to produce lower acuity estimates in the majority of children their advantage is that obtaining any threshold from the severely intellectually impaired provides useful information. It has been proposed that mentally handicapped children with visual impairment are inappropriately classed as profoundly handicapped more often than sighted children with equivalent levels of mental handicap. ${ }^{25}$ A measurement of visual function is therefore important in these children to quantify the visual impairment to provide maximum potential for learning.

The Cardiff and Keeler acuity cards showed very close agreement in those handicapped children assessed with both. Cardiff cards were developed to examine toddlers and older children, who quickly become bored with stripes, and our impression was that the more able children enjoyed these cards and completed testing more quickly. The different style of targets between the two sets of cards did not have any effect on the thresholds achieved and the agreement between the cards was independent of the acuity measured. Due to the small numbers of children in the group that was assessed we would not conclude that two sets of cards are interchangeable. However, this relationship will be further investigated in larger study groups.

Comparison of acuities determined by VEPs with those from acuity cards in the present study shows that there is a significant relationship between thresholds achieved. However, VEP acuities are lower than acuity card acuities in the majority of cases. The use of signal reproducibility as the criterion for threshold is likely to produce more conservative threshold estimates than other commonly used criteria, which produce VEP acuities higher than acuity cards acuities. ${ }^{4}$ Threshold differences between the two techniques may occur as they are testing two different types of visual responses. Preferential looking is a behavioural response; the patient chooses to look at the stimulus. This requires both intact visual input and resolution, and motor output. The VEP records activity of the visual cortex up to area 17, but does not require perception of the stimulus by higher centres. The response requires fixation, but no motor output. In multiply handicapped children damage to the brain is often diffuse, and it is possible that patients may be capable of a response to one type of test but unable to respond to another, regardless of their acuity.

In conclusion, acuity card and VEP techniques both have limitations when assessing vision in multiply handicapped children. Clinically the most information will be obtained by performing both tests on each child and using the information from each test in a complementary manner to provide an overall picture of each child's visual impairment.

The authors thank Michael Bradnam, Aled Evans and Martin Glegg of the West of Scotland Health Boards Department of Clinical Physics for development of the VEP testing system. This research is funded by the Chief Scientist's Office, Disability Research Committee, Scottish Office Home and Health Department (K/RED/4/C183). Roisin Mackie is the current Elizabeth Murray Memorial scholar.

Key words: Acuity cards, Multiple handicap, Visual acuity, Visual evoked potentials.

\section{REFERENCES}

1. van der Pol BA. Causes of visual impairment in children. Doc Ophthalmol 1986;61:223-8.

2. Hertz BG, Rosenberg J. Acuity card testing of spastic children: preliminary results. J Ped Ophthalmol Strabismus 1988;25:139-44.

3. Mohn G, van Hof-van Duin J, Fetter WP, de Grot L, Hage M. Acuity assessment of non-verbal infants and children: clinical experience with the acuity card procedure. Dev Med Child Neurol 1988;30:232-44.

4. Orel-Bixler D, Haegerstrom-Portnoy G, Hall A. Visual assessment of the multiply handicapped patient. Optom Vis Sci 1989;66:530-6.

5. Jacobson L. Ophthalmology in mentally retarded adults. Acta Ophthalmol 1988;66:457-62.

6. Scheimann M. Optometric findings in children with cerebral palsy. Am J Optom Physiol Opt 1984; 61:321-3.

7. Kennerly Bankes JL. Eye defects of mentally handicapped children. BMJ 1974;2:533-5.

8. Lyle W, Woodruff ME, Zuccaro VS. A review of the literature on Down's syndrome and an optometrical survey of 44 patients with the syndrome. Am J Optom Arch Am Acad Optom 1972;49:715-27.

9. Woodruff ME, Cleary TE, Bader D. The prevalence of refractive and ocular anomalies among 1242 institu- 
tionalised mentally retarded persons. Am J Optom Physiol Opt 1980;57:70-84.

10. Black P. Visual disorders associated with cerebral palsy. Br J Ophthalmol 1982;66:46-52.

11. LoCascio GP. A study of vision in cerebral palsy. Am J Optom Physiol Opt 1977;54:332-7.

12. Morante A, Dubowitz LMS, Levene M, Dubowitz V. The development of visual function in normal and neurologically abnormal preterm and fullterm infants. Dev Med Child Neurol 1982;24:771-84.

13. Duckman RH, Selenow A. Use of FPL for measurement of visual acuity in a population of neurologically impaired children. Am J Optom Physiol Opt 1983; 60:817-21.

14. Mayer DL, Fulton AB, Sossen P. Preferential looking acuity of pediatric patients with developmental disabilities. Behav Brain Res 1983;10:189-98.

15. Dubowitz LMS, Mishin J, Morante A, Placzek M. The maturation of visual acuity in neurologically normal and abnormal newborn infants. Behav Brain Res 1983; 10:39-45.

16. Jenkins PL, Simon JW, Gillray LK, Forster T. A simple grating visual acuity test for impaired children. Am J Ophthamol 1985;99:652-8.

17. Mohn G, van Hof-van Duin J. Rapid assessment of visual acuity in infants and children in a clinical setting, using acuity cards. Doc Ophthalmol 1986;45:363-72.
18. Hertz BG, Rosenberg J. Effect of mental retardation and motor disability on testing with visual acuity cards. Dev Med Child Neurol 1992;34:115-22.

19. Mohn G, van Hof-van Duin J. Preferential looking acuity in normal and neurologically abnormal infants and pediatric patients. Doc Ophthalmol 1986;45 221-30.

20. Mohn G, van Hof-van Duin J. Behavioural and electrophysiological measures of visual functions in children with neurological disorders. Behav Brain Res 1983;10:177-87.

21. McCulloch DL, Skarf B. Development of the human visual system: monocular and binocular pattern VEP latency. Invest Ophthalmol Vis Sci 1991;32:2372-81.

22. Woodhouse MJ, Adoh T, Oduwaiye K, Batchelor BG, Megji S, Unwin N, Jones N. New acuity test for toddlers. Ophthalmic Physiol Opt 1992;12:249-51.

23. Robinson J, Mosley MJ, Fielder AR. Grating acuity cards: spurious resolution and the 'edge artefact'. Clin Vis Sci 1988;3:285-8.

24. Dobson V, Teller DY. Visual acuity in human infants; a review and comparison of behavioural and electrophysiological studies. Vision Res 1978;18:1469-83.

25 . Warburg $\mathbf{M}$. Why are the blind and severely visually impaired children with mental retardation much more retarded than the sighted children? Acta Ophthalmol (Suppl) 1982;157:72-8. 